\title{
An electric-field induced dynamical state in dispersions of highly charged colloidal rods: comparison of experiment and theory
}

\author{
K. Kang ${ }^{1}$ J. K. G. Dhont ${ }^{1,2}$
}

Received: 29 April 2015 / Revised: 8 July 2015 / Accepted: 14 July 2015 / Published online: 9 August 2015

(C) The Author(s) 2015. This article is published with open access at Springerlink.com

\begin{abstract}
Concentrated dispersions of highly charged rodlike colloids (fd-virus particles) in isotropic-nematic coexistence exhibit a dynamical state when subjected to lowfrequency electric fields [Soft Matter, 2010, 6, 273]. This dynamical state consists of nematic domains which persistently melt and form on time scales typically of the order of seconds. The origin of the dynamical state has been attributed to a field-induced, cyclic dissociation and association of condensed ions [Soft Matter, 2014, 10, 1987, Soft Matter, 2015, 11, 2893]. The ionic strength increases on dissociation of condensed ions, rendering the nematic domains unstable, while the subsequent decrease of the ionic strength due to association of condensed ions leads to a recurrent stabilization of the nematic state. The role of dissociation/association of condensed ions in the phase/state behaviour of charged colloids in electric fields has not been addressed before. The electric field strength that is necessary to dissociate sufficient condensed ions to render a nematic domain unstable, depends critically on the ambient ionic strength of the dispersion without the external field, as well as the rod-concentration. The aim of this paper is to compare experimental results for the location of transition lines and the dynamics of melting and forming of nematic domains at various ionic strengths and rod-concentrations
\end{abstract}

K. Kang

k.kang@fz-juelich.de

1 Soft Condensed Matter, Forschungszentrum Jülich Institute of Complex Systems (ICS-3), D-52425 Jülich, Germany

2 Department of Physics, Heinrich-Heine Universität Düsseldorf, D-40225 Düsseldorf, Germany with the ion-dissociation/association model. Phase/state diagrams in the field-amplitude versus frequency plane at two different ambient ionic strengths and various rodconcentrations are presented, and compared to the theory. The time scale on which melting and forming of the nematic domains occurs diverges on approach of the transition line where the dynamical state appears. The corresponding critical exponents have been measured by means of image time-correlation spectroscopy [Eur. Phys. J. E, 2009, 30, 333], and are compared to the theoretical values predicted by the ion-dissociation/association model.

Keywords Colloids · Electric fields · Dynamical state · Nematic $\cdot$ Fd-virus

\section{Introduction}

Phase transitions in colloidal systems can be induced by external electric fields due to interactions between polarization charges. At high frequencies (in the $\mathrm{MHz}$ range), dielectric polarization of the cores of spherical colloids give rise to the formation of strings of particles, and their subsequent field-assisted assembly into sheets [1-4]. At these high frequencies, the structure of the diffuse electric double layer is not affected by the electric field. In addition, hydrodynamic interactions between the colloidal particles through field-induced electro-osmotic flow ceases to occur at these high frequencies. For lower frequencies, below about a kHz, interactions between colloids are induced through the polarization of electric double layers and electro-osmotic flow. These two types of field-induced interactions give rise to phase transitions, dynamical states, and pattern formation. The coupling between polarization of the diffuse electric double layers and field-induced electro-osmotic flow is, 
for example, responsible for the formation of mesoscopically large zig-zag structures, where electro-osmotic flow persists along the boundaries of the zig-zag structures [57]. The present authors found various phases and states that are induced by similarly low-frequency electric fields in dispersions of highly charged colloidal rods, at a concentration where there is isotropic-nematic coexistence in the absence of the electric field [8]. In particular, a fieldinduced dynamical state is found where nematic domains persistently melt and form on a seconds time scale. A mechanism underlying the existence of this dynamical state is proposed in Ref. [9], where temporal cyclic dissociation and association of condensed ions plays an essential role. The dissociation of condensed ions into the bulk dispersion leads to a decreased Debye screening length which can destabilize the nematic. After melting of the nematic, reassociation of condensed ions occurs, leading to a decreased Debye length and a re-stabilization of the nematic state. Such a mechanism of dissociation and association of condensed ions has not been considered before to play a role in field-induced states. Although the location of state transition lines in the field amplitude versus frequency plane for two rod-concentrations can be described by the iondissociation/association model for a fixed ionic strength (as shown in Ref. [9], with a corrigendum in Ref.[10]), the critical test where also the ambient ionic strength is varied has not been performed yet. According to the iondissociation/association model, there should be a strong dependence of the location of transition lines on the ambient ionic strength. In addition, we present here a comparison with experimental results on the dynamics of melting and forming of nematic domains from Ref. [11], with an emphasis on the critical divergence of the corresponding time scales on approach of the transition line. The aim of this paper is therefore to test the dissociation/association model as presented in Refs. [9, 10] against experiments where phase/state diagrams and time scales for melting and forming of nematic domains are measured at various ionic strengths, as well as various rod-concentrations.

As a model system for rod-like colloids, we use fd-virus particles. These particles have been used in the past as colloidal model systems for the study of the isotropic-nematic phase transition under the influence of a magnetic field [12], and later for extensive studies of various liquid crystalline phases without an external field [13-20], as well as for single-particle diffusive behaviour [21-23]. In particular, a chiral nematic phase is found for ionic strengths down to $5 \mathrm{mM}[13,14,18,19]$, which is attributed to the helical core-structure of the fd-virus. Fd-viruses consist of a DNA strand with a contour length of $880 \mathrm{~nm}$, which is covered by 2700 proteins. The diameter of the core is $6.8 \mathrm{~nm}$, while the coat proteins renders the fd-viruses relatively stiff, with a persistence length of about $2500 \mathrm{~nm}$. At a $\mathrm{pH}$ around 7, the coat proteins carry 8800 negative elementary charges [24], which leads to strong Manning-ion-condensation, where the majority of the immobile surface charges are neutralized by condensed ions.

This paper is organized as follows. In "The electric phase/state diagram" section, we briefly present the general features of the phase/state diagram. The theory that semiquantitatively describes the location of the transition line in the field-amplitude versus frequency plane to the dynamical state, as well as the dynamical features of melting and forming of nematic domains, is summarized in "The ion-dissociation/association model" section. A necessary ingredient of the theory is the lower isotropic-nematic binodal concentration, which is addressed in the "Binodal and spinodal concentrations" section. In the "Comparison to experiments" section, we present experimental phase/state diagrams for two buffer concentrations of 0.16 and $0.0032 \mathrm{mM}$, corresponding to two different ionic strengths, and for various fd-concentrations. The location of the transition line to the dynamical state is compared to the prediction by the dissociation/association model in "The location of the $N^{*}$-to- $D$ transition line" section. The dynamics of melting and forming of nematic domains is discussed in "The dynamical behavior on approach of the $N^{*}$-to- $D$ transition line" section.

\section{The electric phase/state diagram}

The phase/state diagram in the field strength versus frequency plane is given in Fig. 1, for a fd-concentration of $2.0 \mathrm{mg} / \mathrm{ml}$. The fd-dispersion is dialyzed against a TRIS/HCl buffer solution with a concentration of $0.032 \mathrm{mM}$, with a $\mathrm{pH}$ of 5.8 . The following phases and states are found (for a more detailed description of the various phases and states, see Ref. [8]):

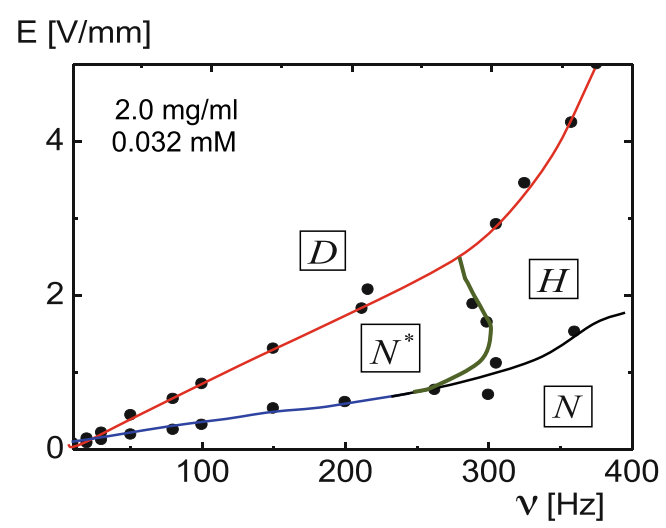

Fig. 1 The phase/state diagram for a fd-concentration of $2.0 \mathrm{mg} / \mathrm{ml}$ and a TRIS/HCl-buffer concentration of $0.032 \mathrm{mM}$. The various phases are discussed in the main text 
(i) The $N$-phase is a coexistence between isotropic and (non-chiral) nematic regions. This is the phase that exists without the external electric field, and persist to be stable up to a finite electric field strength. As mentioned in the introduction, at buffer concentrations larger than $5 \mathrm{mM}$, the nematic is chiral, due to the helical structure of the core of fd-virus particles. For the low ambient ionic strength used here, the long-ranged electrostatic repulsions render the average distance between fd-rods sufficiently large, so that the helical structure of the cores is screened. The Debye length at this buffer concentration is equal to $54 \mathrm{~nm}$ (where the solution of carbon dioxide from the air is taken into account [25]).

(ii) For frequencies below about $300 \mathrm{~Hz}$, the nematic domains become chiral nematic, the $N^{*}$-phase, on increasing the field amplitude. The $N$-to- $N^{*}$ transition line is indicated in blue in Fig. 1. This transition is most probably due to the increase of the ionic strength resulting from the overall dissociation of condensed ions. The chiral nematic state is also seen without the electric field but for larger ambient ionic strengths.

(iii) At frequencies larger than about $300 \mathrm{~Hz}$, a uniform phase is found, where the rods are aligned along the electric field, perpendicular to the electrodes. This phase is named the $H$-phase, standing for the homeotropic alignment of the rods. The frequency at which the polarization of the electric double layer and the layer of condensed ions ceases to occur can be estimated to be around the frequency where the $N^{*}$-to- $H$ transition line is located (the green line in Fig. 1). The $H$-phase is therefore stabilized by hydrodynamic interactions through the field-induced electro-osmotic flow. (iv) At sufficiently high electric field strengths (above the red line in Fig. 1), the $N^{*}$ - and $H$-phase transform to a dynamical state, the $D$-state, where nematic domains persistently melt and form.

There are two additional gradual transitions which are not indicated Fig. 1. Within the $N^{*}$-phase, at low field strengths, the nematic domains form a seemingly interconnected structure. On increasing the electric field strength, the interconnectivity is gradually lost. There is thus a gradual transition within the $N^{*}$-phase where the chiral nematic texture becomes disconnected on increasing the field strength [8]. Close to the $N^{*}$-to- $D$ transition line the dynamics of melting is very slow, while far from the transition line, the time scale on which melting and forming of domains occurs levels off to about $1.5 \mathrm{~s}$. There is thus a gradual transition from very slow to fast dynamics of melting and forming of nematic domains.

It is the dynamical state that is of interest in this paper. In the next section, the microscopic origin of this state is discussed, first on an intuitive level, followed by a semiquantitative analysis.

\section{The ion-dissociation/association model}

The microscopic origin for the existence of the $D$-state is attributed to the cyclic dissociation and association of condensed ions. Figure 2, which is taken from Ref. [9], illustrates the mechanism through which such a cyclic dissociation and association is kinetically induced. First consider a nematic domain with its director aligned along the direction of the electric field (stage (I) in Fig. 2). In Fig. 2, only two rods out of the entire domain are depicted for clarity. The domain orientation along the field direction
Fig. 2 A sketch of the microscopic origin of the dynamical state, where nematic domains melt and form. The different stages during a cycle of melting-and-forming of a domain are discussed in the main text

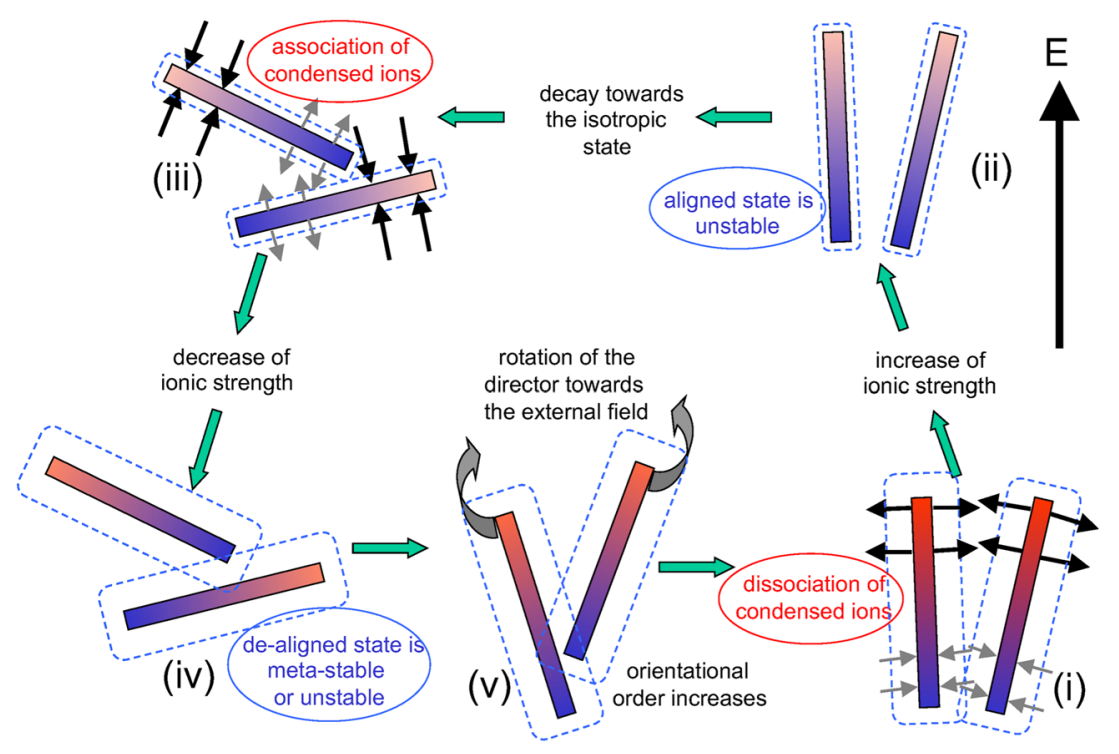


is a consequence of single-particle torques resulting from field-induced polarization of the double-layer and the layer of condensed ions. In such an orientation, their is a relatively large amount of excess condensed (positive) ions at the top side of the rod (indicated in red), while there is a depleted region at the bottom part (indicated in blue). The excess of condensed ions at the top creates an electric field that pushes the ions out-of the condensed layer into bulk solution (indicated by the arrows in stage (I)). The opposite happens at the bottom part of the rod. As the concentration of ions in the condensed layer is much larger than in the diffuse double layer, the net result is a dissociation of condensed ions. After a time comparable to the time needed for the released ions to diffuse into the bulk solvent, the ionic strength will increase. This will decrease the Debye length (the extent of the diffuse double layer is indicated in Fig. 2 by the dotted blue lines around the core of the rods). Hence, in stage (II), the rods carry less condensed ions, while the Debye length is smaller. A reduction of the Debye length corresponds to a reduction of the effective concentration (the effective concentration will be quantified in the subsequent paragraph). When the effective concentration becomes less than the lower isotropic-nematic binodal, the nematic domain becomes unstable and melts, which is accompanied by a de-alignment, as depicted in Fig. 2 in stage (III). Association of condensed ions occurs as the rods take orientations towards directions perpendicular to the electric field. This leads in turn to a decreased ambient ionic strength, and thereby to an increase of the Debye length (as depicted in stage (IV)). The accompanied increase of the effective concentration renders the nematic phase stable again. The resulting nematic domain aligns along the electric field direction due to single-particle torques (see stage (V)), during which polarization takes place leading to stage (I), after which the cycle subsequently repeats itself.

The above-mentioned time-dependent "effective concentration" is to be understood as follows. For suspensions of very long and thin colloidal rods with hard-core interactions, Onsager showed that the location of isotropic-nematic binodal- and spinodal concentrations depends on the dimensionless concentration $(L / d) \varphi$, with $L$ the length of the rod, $d$ the core diameter, and $\varphi=(\pi / 4) d^{2} L \rho$ the volume fraction (the fraction of the total volume occupied by the cores of the rods), with $\rho$ the number concentration of rods $[26,27]$. In case the rods are charged, the same Onsager theory can be employed, except that the core thickness is larger due to the additional repulsive electrostatic interactions. This defines an effective, time-dependent diameter $d_{e f f}$, and thereby an effective dimensionless concentration $\left(L / d_{e f f}\right) \varphi_{e f f}$. The following expression for the effective diameter can be derived $[9,10]$,

$d_{e f f}=\kappa^{-1}\left[\ln K_{Q}+\gamma_{E}\right]$, where $\kappa^{-1}$ is the Debye length and $\gamma_{E}=0.5772 \ldots$ is Euler's constant, and where,

$K_{Q}=\frac{2 \pi \exp \{\kappa d\}}{\left(1+\frac{1}{2} \kappa d\right)^{2}} \frac{l_{B}}{\kappa L^{2}}\left(N_{0}-N_{c, 0}\right)^{2}$,

with $d$, as before, the core diameter, $l_{B}$ is the Bjerrum length, $N_{0}$ is the number of immobile charges chemically attached to the surface of a rod, and $N_{c, 0}$ the number of condensed ions of a rod in the absence of an electric field. Considerations concerning the quantification of an effective diameter can also be found in Refs. [26-30]. Note that the total rod-surface charge that is relevant for the ionconcentrations within the diffuse double layer is equal to $-e\left(N_{0}-N_{c, 0}\right)$ (with $e$ the elementary charge), which the total immobile surface-charge plus the total charge within the layer of condensed ions.

In order to describe the dynamics of melting and forming of nematic domains, an equation of motion for the orientational order parameter tensor $\mathbf{S}$ should be derived. This tensor is defined as the ensemble average of the dyadic product of the unit vector $\hat{\mathbf{u}}$ that specifies the orientation of a rod,

$\mathbf{S}(t) \equiv<\hat{\mathbf{u}} \hat{\mathbf{u}}>(t)$.

The largest eigenvalue $\lambda$ of the orientational order parameter tensor quantifies the degree of alignment of the rods. In the isotropic phase $\lambda=1 / 3$ while in a perfectly aligned state $\lambda=1$. The concentration dependence of the order parameter (without the electric field) is most conveniently understood on the basis of the bifurcation diagram given in Fig. 3. The values of the effective concentration $\left(L / d_{\text {eff }}\right) \varphi_{\text {eff }}$ where the isotropic-nematic binodals and spinodals are located, according to Onsager [26, 27], are given on the lower axis of the bifurcation diagram. Here, $C_{b i n}^{(-)}$and $C_{b i n}^{(+)}$are the lower and upper binodal concentrations. For initial overall concentrations in between the two binodal concentrations, the equilibrium state is a coexistence between an isotropic and nematic phase. For concentrations below $C_{b i n}^{(-)}$, the isotropic phase is stable, and above $C_{b i n}^{(+)}$the nematic phase is stable. The spinodal concentrations $C_{\text {spin }}^{( \pm)}$relate to the stability of the uniform isotropic and nematic state. The spinodal concentration $C_{\text {spin }}^{(+)}$marks the concentration where a uniform isotropic state becomes unstable against the uniform nematic state upon increasing the concentration. The vertical dashed arrow in Fig. 3 depicts the temporal increase of the orientational order parameter towards the nematic branch (the solid line in blue). On lowering the concentration of a uniform nematic below the spinodal concentration $C_{\text {spin }}^{(-)}$, the nematic becomes unstable against the isotropic state. The order parameter of the uniform nematic state now decreases 


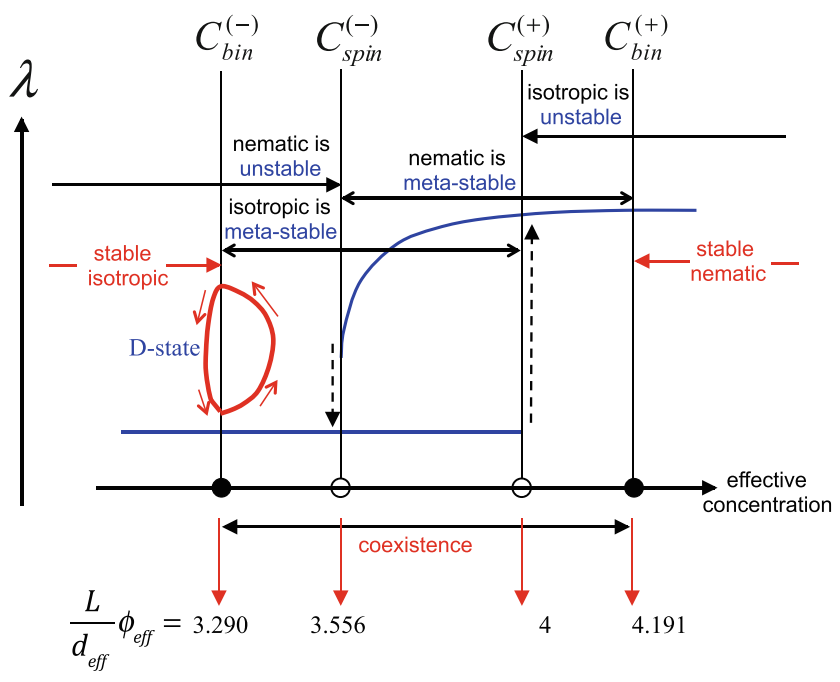

Fig. 3 A sketch of the bifurcation diagram for the isotropic-nematic phase transition, where the orientational order parameter $\lambda$ is plotted against the effective concentration. The location of binodals, spinodals, and stability curves are given, which bound regions where the isotropic and nematic state are either unstable or meta-stable. The Onsager values of the effective concentration for the location of binodals and spinodals are given in the lower part of the figure. The red, closed curve is a sketch of the limit cycle, indicating cyclic melting and forming of nematic domains

towards $1 / 3$. We thus find that the uniform nematic is unstable for effective concentrations $\left(L / d_{\text {eff }}\right) \varphi_{\text {eff }}<C_{\text {spin }}^{(-)}$, while the uniform isotropic state is meta-stable for concentrations $\left(L / d_{e f f}\right) \varphi_{\text {eff }}<C_{\text {spin }}^{(+)}$, as indicated in Fig. 3. The red, closed curve depicts the limit cycle corresponding to the alternating crossing of the lower binodal in the dynamical state under the action of an electric field. Hence, melting of the nematic state occurs from the unstable state, through spinodal decomposition. The isotropic state grows from the meta-stable state, through nucleation and growth. Nucleation times in the present case are probably small, as there is some reminiscent alignment after melting.

In order to quantify the dynamics of melting and forming of nematic domains in the dynamical state, according to the above discussion, we need two equations of motion for the orientational order parameter tensor (2): one equation of motion for spinodal melting of the nematic state when $\left(L / d_{e f f}\right) \varphi_{\text {eff }}<C_{\text {bin }}^{(-)}$, and one for nucleation and growth of the nematic from an (near-) isotropic state when $\left(L / d_{e f f}\right) \varphi_{\text {eff }}>C_{\text {bin }}^{(-)}$.

An equation of motion for spinodal decomposition of a nematic state can be derived from the Smoluchowski equation, through a Ginzburg-Landau expansion upto fourth order in the orientational order parameter. Such a GinzburgLandau expansion can only be employed to describe the kinetics of an initially unstable state, and therefore describes melting of the nematic, as discussed above. For frequencies of the external field that are sufficiently large that during a cycle of the field the configuration of the rods is essentially unchanged (for the fd-suspensions under consideration, this frequency is about $50-100 \mathrm{~Hz}$ ), an equation for the orientational order parameter tensor can be derived from the Smoluchowski equation $[9,10]$. This equation of motion can be written as a sum of various contributions,

$$
\frac{\partial \mathbf{S}}{\partial \tau}=\Delta_{i d}+\Delta_{Q, h c}+\Delta_{t w i s t}+\Delta_{p o l}+\Delta_{\text {torque }} \text {, }
$$

with the dimensionless time variable,

$\tau=D_{r} t$

where $D_{r}$ is the free rotational diffusion coefficient. The various contributions are as follows. First of all, $\Delta_{i d}$ is the contribution from free diffusion,

$\Delta_{i d}=6\left[\frac{1}{3} \hat{\mathbf{I}}-\mathbf{S}\right]$.

The second contribution $\Delta_{Q, h c}$ stems from interactions, unperturbed by the external field, with an effective hard-core diameter that accounts for the above discussed electrostatic interactions, as indicated by the subscript " $Q$ ",

$\Delta_{Q, h c}=\frac{9}{2} \frac{L}{d_{e f f}} \varphi_{e f f}\{\mathbf{S} \cdot \mathbf{S}-\mathbf{S} \mathbf{S}: \mathbf{S}\}$.

The third contribution $\Delta_{\text {twist }}$ is the twist contribution,

$\Delta_{t w i s t}=-\frac{9}{2}\left[\frac{5}{4}-\ln 2\right] \frac{1}{\kappa d_{e f f}} \frac{L}{d_{e f f}} \varphi_{e f f}\{\mathbf{S} \cdot \mathbf{S}-\mathbf{S} \mathbf{S}: \mathbf{S}\}$.

This contribution describes the effect, referred to as "the twist effect" $[28,29]$, that there is a preference for a non-parallel, twisted orientation of two rods due to the energetically unfavorable overlap of diffuse double layers in parallel orientation. The contribution $\Delta_{p o l}$ is the contribution due to interactions from polarization charges,

$\Delta_{p o l}=\frac{7}{60}\left[\frac{K_{E}}{K_{Q}}\right]^{2} \frac{1}{\kappa d_{e f f}} \frac{L}{d_{e f f}} \varphi_{e f f} h(\Omega) \mathcal{E}_{0}^{4}\left(\mathbf{S}: \hat{\mathbf{E}}_{0} \hat{\mathbf{E}}_{0}\right) \mathbf{F}\left(\mathbf{S}, \hat{\mathbf{E}}_{0}\right)$,

while $\Delta_{\text {torque }}$ accounts for single-particle torques with which the external field acts on polarization charges,

$\Delta_{\text {torque }}=\frac{1}{80} \frac{L}{l_{B}} \tilde{F} I(\Omega) \mathcal{E}_{0}^{2} \mathbf{F}\left(\mathbf{S}, \hat{\mathbf{E}}_{0}\right)$,

where $\mathcal{E}_{0}$ is the dimensionless external field strength (with $\beta=1 / k_{B} T$, and $E_{0}$ the external field strength),

$\mathcal{E}_{0}=\beta e L E_{0}$,

and $\mathbf{F}\left(\mathbf{S}, \hat{\mathbf{E}}_{0}\right)$ is an abbreviation for,

$$
\begin{aligned}
\mathbf{F}\left(\mathbf{S}, \hat{\mathbf{E}}_{0}\right) \equiv & \frac{3}{2} \mathbf{S} \cdot \hat{\mathbf{E}}_{0} \hat{\mathbf{E}}_{0}+\frac{3}{2} \hat{\mathbf{E}}_{0} \hat{\mathbf{E}}_{0} \cdot \mathbf{S}+\mathbf{S} \cdot \mathbf{S} \cdot \hat{\mathbf{E}}_{0} \hat{\mathbf{E}}_{0} \\
& +\hat{\mathbf{E}}_{0} \hat{\mathbf{E}}_{0} \cdot \mathbf{S} \cdot \mathbf{S}-2 \mathbf{S} \cdot \hat{\mathbf{E}}_{0} \hat{\mathbf{E}}_{0} \cdot \mathbf{S}-3 \mathbf{S ~ S}: \hat{\mathbf{E}}_{0} \hat{\mathbf{E}}_{0},
\end{aligned}
$$

with $\hat{\mathbf{E}}_{0}$ is the unit vector in the direction of the external 
field. The frequency-dependent functions appearing in the above equations are,

$h(\Omega)=\left[\frac{1}{\Omega} \frac{\sin \{\Omega\}+\sinh \{\Omega\}}{[\cos \{2 \Omega\}+\cosh \{2 \Omega\}]^{2}}\right]^{2}\left[1+\frac{4}{3} \Omega^{4}+\frac{2}{5} \Omega^{8}\right]$,

$I(\Omega)=\frac{1}{2 \Omega^{3}} \frac{\sinh \{2 \Omega\}-\sin \{2 \Omega\}}{\cosh \{2 \Omega\}+\cos \{2 \Omega\}}$,

where [31],

$\Omega=\sqrt{\frac{\omega L^{2}}{8 D_{e f f}}}$,

is a dimensionless frequency, with $\omega$ the frequency of the external field, and with $D_{e f f}$ the effective translational diffusion coefficient of the condensed ions, which is equal to,

$D_{\text {eff }}=D\left[1+2 \kappa_{c} a \mathcal{K}(\kappa a)\right]$,

where $D$ is the free translational diffusion coefficient of condensed ions. The second term within the square brackets accounts for the repulsive interactions between the condensed ions, with,

$\kappa_{c}=\frac{2 l_{B}}{d L} N_{c}$,

the inverse "condensate length." The frequency-dependent functions $h(\Omega)$ and $I(\Omega)$ are essentially zero for $\Omega>3$, for which the polarization of the layer of condensed ions ceases to occur. At that frequency, the $H$-phase becomes the stable phase, as discussed in "The electric phase/state diagram" section. The constants $K_{E}$ and $\tilde{F}$ are equal to [9, 10, 31],

$$
\begin{aligned}
K_{E}= & \frac{\pi \exp \{\kappa d\}}{2(1+\kappa a)^{2}\left(1+2 k_{c} a \mathcal{K}(\kappa a)\right)^{2}} \frac{l_{B}}{\kappa L^{2}} N_{c}^{2}, \\
\tilde{F}= & V\left(\kappa_{c} a\right)\left[W\left(\kappa_{c} a, \kappa a\right)+1\right] \\
& \times\left\{2\left[1+\kappa_{c} a \mathcal{B}(\kappa a)\right]^{2}-\kappa_{c} a\left[1+\kappa_{c} a \mathcal{B}(\kappa a)\right]\right\},
\end{aligned}
$$

with $a=d / 2$ the hard-core radius, and where $V$ and $W$ stand for,

$$
\begin{aligned}
V\left(\kappa_{c} a\right) & =\frac{\kappa_{c} a}{\left(1+\kappa_{c} a \mathcal{B}(\kappa a)\right)^{2}}, \\
W\left(\kappa_{c} a, \kappa a\right) & =-\frac{2 \kappa_{c} a \mathcal{K}(\kappa a)}{1+2 \kappa_{c} a \mathcal{K}(\kappa a)},
\end{aligned}
$$

with $\left(K_{0}\right.$ is the modified Bessel function of the second kind of zeroth order),

$$
\begin{aligned}
& \mathcal{K}(\kappa a) \equiv \frac{1}{2 \pi} \int_{0}^{2 \pi} d \varphi K_{0}(\kappa a \sqrt{2(1-\cos \varphi)}), \\
& \mathcal{B}(\kappa a) \equiv \frac{1}{\pi} \int_{0}^{2 \pi} d \varphi \cos \{\varphi\} K_{0}(\kappa a \sqrt{2(1-\cos \varphi)}) .
\end{aligned}
$$

An equation of motion for the nucleation and growth of a nematic domain from the isotropic state requires the solution of the Smoluchowski equation including all orders of the orientational order parameter, as well as the spatial dependence of the orientational order parameter tensor. This is a problem that is probably too complicated to allow for an analytical treatment. We therefore adopt the exponential growth that is found in simulations [32],

$\frac{\partial \mathbf{S}}{\partial \tau}=\frac{\overline{\mathbf{S}}-\mathbf{S}}{\mathcal{T}}+\Delta_{\text {pol }}+\Delta_{\text {torque }}$,

where $\overline{\mathbf{S}}$ is the order parameter tensor of the nematic phase in equilibrium, without the electric field, and where $\mathcal{T}$ is the time scale on which the internal orientational order of domains increases. The last term is responsible for the orientation of the nematic director towards the electric-field direction, which is an essential ingredient for the existence of the dynamical state.

We note that both equations of motion $(3,6)$ neglect the finite size of nematic domains. Spatial variation of the orientational order parameter is not considered here. This is probably a reasonable approximation in view of the quite fuzzy interface between the nematic and isotropic regions, as evidenced from microscopy images. On the other hand, the dynamics of a given domain might be affected by adjacent domains.

An oscillatory state is only found when dissociation/association of condensed ions is included. In Ref. [9], the following semi-empirical equation of motion for the number $N_{c}$ of condensed ions is proposed,

$$
\begin{aligned}
\frac{d N_{c}}{d \tau}= & \pm \mathcal{C}_{d}\left\{N_{c}^{2}-N_{\text {lim }}^{2}\right\}\left(\frac{z^{2} l_{B}}{L\left[1+2 \kappa_{c} a \mathcal{K}(\kappa a)\right]}\right)^{2} \\
& \times \mathcal{E}_{0}^{2}\left(\hat{\mathbf{E}}_{0} \hat{\mathbf{E}}_{0}:\left[\mathbf{S}(t)-\alpha_{t h r} \hat{\mathbf{I}}\right]\right) I(\Omega),
\end{aligned}
$$

where $C_{d}$ is the "effective dissociation constant." Dissociation occurs only when there is sufficient polarization of the layer of condensed ions along the long axis of the rod, which requires a minimum component of the orientation of a rod along the external field. The number $\alpha_{t h r}$ thus specifies the minimum value of the orientation along the field direction upon which dissociation can occur. When $\left(\mathbf{S}: \hat{\mathbf{E}}_{0} \hat{\mathbf{E}}_{0}\right.$ ) $>\alpha_{t h r}$ dissociation occurs (and the "- " in Eq. 7 applies), whenever the actual number of condensed ions is larger than the limiting number of condensed ions $N_{\text {lim }}$, which is given by,

$$
N_{l i m}=\frac{\alpha_{l i m} N_{c, 0}}{\alpha_{l i m}+\mathcal{E}_{0}^{2}\left(\hat{\mathbf{E}}_{0} \hat{\mathbf{E}}_{0}: \mathbf{S}\right) I(\Omega)}
$$

when, $\quad\left(\mathbf{S}: \hat{\mathbf{E}}_{0} \hat{\mathbf{E}}_{0}\right)>\alpha_{t h r}$.

This is the limiting, time averaged number of condensed ions in the stationary state when a rod with a fixed orientation is subjected to the external field for a long time. When, on the other hand, $\left(\mathbf{S}: \hat{\mathbf{E}}_{0} \hat{\mathbf{E}}_{0}\right)<\alpha_{t h r}$ (and the "+" applies), association of condensed ions occurs, and,

$N_{\text {lim }}=N_{c, 0}, \quad$ when, $\quad\left(\mathbf{S}: \hat{\mathbf{E}}_{0} \hat{\mathbf{E}}_{0}\right)<\alpha_{t h r}$, 
where, as before, $N_{c, 0}$ is the number of condensed ions in the absence of the external field (note that $N_{\text {lim }} \leq N_{c, 0}$ ).

It takes some time before the ion-concentration within the bulk of the solvent is affected by the dissociation or association of condensed ions. Ions that dissociate from the condensed layer must diffuse over distances of the order of a rod length, in order to change the bulk ionic strength. Similarly, it takes some time for ions to diffuse from the bulk to the condensed layer as association occurs. The change of the bulk concentration of ions at time $t$ is thus approximately proportional to the number $\Delta N_{c}=N_{c, 0}-N_{c}$ of released ions at an earlier time $t-\tau_{\text {dif }}$, where $\tau_{\text {dif }}$ is the time required for ions to diffuse over distances of the order of a rod length. The time-dependent (inverse) Debye length at time $t$ is therefore taken equal to, ${ }^{1}$

$\kappa(t)=\sqrt{\frac{\beta e^{2}\left[2 c_{0}+\bar{\rho} \Delta N_{c}\left(t-\tau_{d i f}\right)\right]}{\epsilon}}$,

where $\bar{\rho}$ is the number density of rods, and $c_{0}$ is the ambient ionic strength,

$c_{0}=\frac{1}{2} \sum_{\alpha} c_{\alpha} z_{\alpha}^{2}$,

where the summation ranges over all species of ions in bulk solution, and $c_{\alpha}$ is the number concentration of species $\alpha$ (with valency $z_{\alpha}$ ), in the absence of the electric field. Therefore, the effective diameter in Eq. 1 becomes timedependent,

$\frac{d_{e f f}(t)}{d}=\frac{1}{\kappa(t) d}\left[\ln \left\{K_{Q}(\kappa \equiv \kappa(t))\right\}+\gamma_{E}\right]$,

where the interaction strength $K_{Q}$ is evaluated with an inverse Debye length equal to $\kappa(t)$. This time dependence quantifies the variation of the effective concentration upon dissociation/association of condensed ions, which is at the origin of the dynamical state.

\section{Binodal and spinodal concentrations}

As a last step, we have to specify the lower binodal concentration $C_{b i n}^{(-)}$in order to decide whether Eq. 3 or 6 should be used to describe the dynamics of the order parameter. It turns out that the external electric field has a very minor effect on the concentration where the isotropicnematic phase transition line is located. The location of the lower binodal concentration, including the twist effect, can therefore simply be obtained from the corresponding the dimensionless concentration $(L / d) \varphi=3.290 \cdots$ for hard-spheres, as predicted by Onsager [26, 27]. This relies on the fact that in the equation of motion (3), the chargecharge interactions as well as the twist effect have the same

\footnotetext{
${ }^{1}$ Note that there is a misprint in Eq. (48) in Ref.[9]
}

functional dependence on the orientational order parameter tensor. The lower binodal concentration is thus set by,

$$
\begin{aligned}
{\left[\frac{L}{d} \varphi\right]_{\text {Onsager }} } & =3.290 \cdots \\
& =\frac{L}{d_{\text {eff }}} \varphi_{\text {eff }}\left\{1-\left[\frac{5}{4}-\ln 2\right] \frac{1}{\kappa d_{\text {eff }}}\right\} .
\end{aligned}
$$

The same procedure can be used to obtain the location of the upper binodal and two spinodal concentrations. The Onsager value of $(L / d) \varphi$ for the upper binodal is 4.191, and for the lower and upper spinodal 3.556 and 4, respectively. The location of binodals and spinodals depend both on the Debye screening length, as well as the effective number of surface charges $N_{0}-N_{c, 0}$ (as before, $N_{0}$ is the total number of immobile bare charges on a single rod, and $N_{c, 0}$ is the number of condensed ions in the absence of the external electric field). The plots in Fig. 4 give the binodal and spinodal concentrations as a function of the Debye length for several values of the effective number of charges.

The predicted isotropic-nematic coexistence regions for $N_{0}-N_{c, 0}=500$ (a number that is found from fits to the location of the $N^{*}$-to- $D$ transition line, as discussed in the next section) are $1.68<[f d]<2.14 \mathrm{mg} / \mathrm{ml}$ and $0.66<[f d]<0.84 \mathrm{mg} / \mathrm{ml}$ for buffer concentrations of

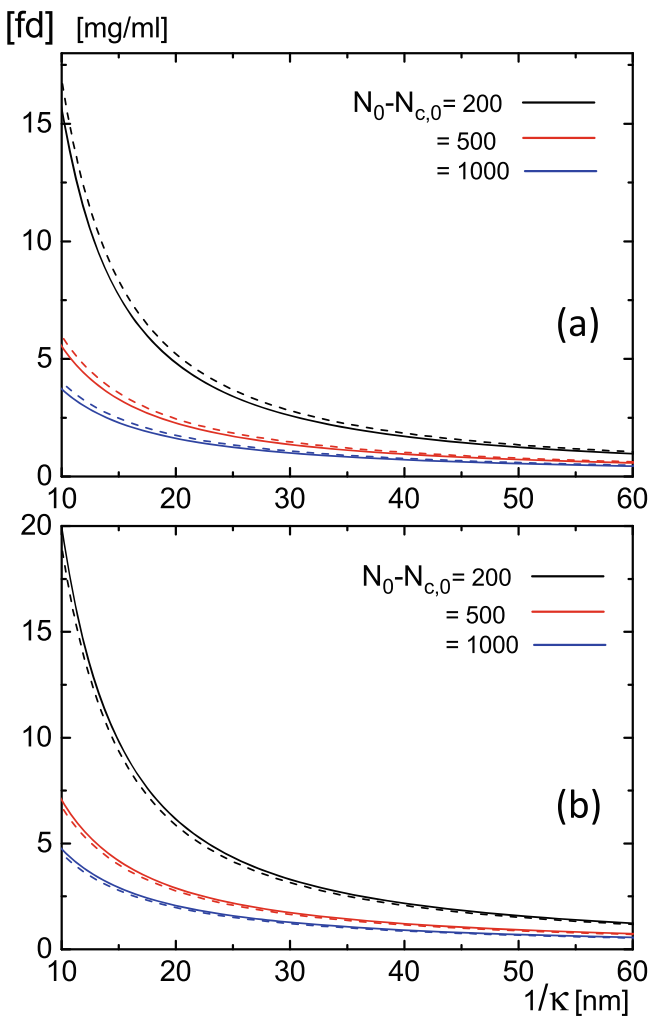

Fig. 4 The lower $\mathbf{a}$ and upper $\mathbf{b}$ binodal and spinodal concentrations, as a function of the Debye length for several values of the number of effective charges $N_{0}-N_{c, 0}$. Dashed lines are spinodals and solid lines are binodals 
0.16 and $0.032 \mathrm{mM}$, respectively. The experimental binodal concentrations are $1.5 \pm 0.2$ and $3.4 \pm 0.5 \mathrm{mg} / \mathrm{ml}$ for the $0.16 \mathrm{mM}$ buffer, and $0.8 \pm 0.2$ and $1.5 \pm 0.4 \mathrm{mg} / \mathrm{ml}$ for the $0.032 \mathrm{mM}$ buffer. The lower binodal concentrations are in very good agreement, while the experimental upper binodal concentrations are slightly higher than the theoretically predicted values.

\section{Comparison to experiments}

In a comparison to experiments, the volume fraction $\varphi$ must be calculated from the weight concentration of fd-virus particles. From the length, thickness, and molecular weight of a fd-virus particle, the relation between the hard-core volume fraction and the weight concentration $[f d]$ in units of $\mathrm{mg} / \mathrm{ml}$ is found to be: $\varphi=0.0011 \times[f d]$. In addition, the dimensionless electric field amplitude (4) is calculated from $\mathcal{E}_{0}=0.096 \times 0.035 \times E_{0}[\mathrm{~V} / \mathrm{mm}]$, where the factor 0.096 accounts for the reduction of the applied field strength $E_{0}$ due to the dielectric polarization of the ITOwater interface. The data that will be shown are corrected for the decrease of the electric field strength in the bulk of the suspensions due to the partial buildup of electric double layers at the electrodes, also known as "electrode polarization" [8]. Electrode polarization is essentially absent for frequencies $v=\omega / 2 \pi$ larger than about 60 and $120 \mathrm{~Hz}$ for the buffer concentrations 0.16 and $0.032 \mathrm{mM}$, respectively.

There are a number of parameters in the dissociation/association model of which the numerical values have to specified. Some of these parameters can be specified independently, while others have to be adjusted to fit experimental data. Table 1 shows the parameters that are determined independently for the two buffer concentrations of 0.032 and $0.16 \mathrm{mM}$. The $\mathrm{pH}$ and ionic strength $c_{0}$ are calculated from the buffer concentrations, including the contribution from carbon dioxide that dissolves from the air, as discussed in Ref. [25]. The Debye length $\kappa^{-1}$ (at $25{ }^{\circ} \mathrm{C}$ ) is calculated from the ionic strength: $\kappa^{-1}[\mathrm{~nm}]=0.304 / \sqrt{c_{0}[M]}$. The number $N_{0}$ of immobile charges on a fd-virus particle depends on the $\mathrm{pH}$ through the dissociation/association of surface groups on the coat proteins, which can be obtained from Ref. [24]. The orientational order parameter within the full nematic state is measured as a function of the buffer concentration in Ref.

Table 1 Independent numerical parameter values for the two buffer concentrations

\begin{tabular}{llllll}
\hline Conc. $(\mathrm{mM})$ & $\mathrm{pH}$ & $c_{0}(\mathrm{mM})$ & $\kappa^{-1}(\mathrm{~nm})$ & $N_{0}$ & $\bar{S}$ \\
\hline 0.032 & 5.8 & 0.032 & 53.7 & 7800 & 0.93 \\
0.16 & 6.9 & 0.143 & 25.4 & 8700 & 0.93 \\
\hline
\end{tabular}

[25], down to a buffer concentration of $0.16 \mathrm{mM}$. Extrapolation of the scalar order parameter to the upper-binodal concentration gives the scalar orientational order parameter that is relevant in Eq. 6. The scalar order parameter is essentially the same for the two buffer concentrations, and is equal to $0.93 \pm 0.02$ (a plot of the scalar order parameter versus the buffer concentration from the data in Ref. [25] shows that the difference of the order parameter for the two buffer concentrations is less than 0.01). The order parameter tensor $\overline{\mathbf{S}}$ in Eq. 6 is thus equal to $0.93 \times \hat{\mathbf{E}}_{0} \hat{\mathbf{E}}_{0}$.

\section{The location of the $N^{*}$-to- $D$ transition line}

The location of the $N^{*}$-to- $D$ transition line is independent of the numerical values of the dynamical parameters $\mathcal{T}$ (which sets the nematic growth rate from the metastable state), $\mathcal{C}_{d}$ (the effective dissociation constant), $\tau_{\text {dif }}$ (the time condensed ions need to diffusive into the bulk), and $\alpha_{t h r}$ (that sets the degree of alignment beyond which dissociation occurs). The three remaining parameters that determine the location of $N^{*}$-to- $D$ transition lines are (i) the number $N_{c, 0}$ of condensed ions in equilibrium, without the electric field, (ii) $\alpha_{\min }$ in Eq. 8, which determines the remaining limiting number of condensed ions on a rod with a fixed orientation as a function of the electric field amplitude and frequency, and (iii) the diffusion coefficient of the condensed ions in Eq. 5. The experimental phasestate diagrams for the buffer concentrations $[b]=0.16$ and $0.032 \mathrm{mM}$ are given in Figs. 5 and 6 , respectively. The solid red lines are the theoretical $N^{*}$-to- $D$ transition lines for the values of $N_{c, 0}, \alpha_{\text {lim }}$, and $D / D_{0}$ as given in Table 2 (with $D_{0}=2.0 \times 10^{-9} \mathrm{~m}^{2} / \mathrm{s}$ the typical value of diffusion coefficients of ions in solution). The reduction of the bare diffusion coefficient is due to additional friction of ions with the core of the rod, which is independent of ion concentration. The grey areas in these plots indicate the range of validity of the present theory: the frequency should be larger than about $50-100 \mathrm{~Hz}$ due to the assumed constant orientation of the rods during a cycle of the external electric field, and should not be larger than about $300 \mathrm{~Hz}$ where polarization is essentially absent and hydrodynamic interactions through electro-osmotic flow become significant.

In particular for the lower ionic strength, there are two counter balancing effects on the location of the $N^{*}$-to$D$ transition line as the fd-concentration is increased. For a given frequency, the transition shifts to a higher field amplitude on increasing the fd-concentration, since the increase of the ionic strength due to the dissociation of condensed ions must be larger, as the concentration is further away from the lower-binodal concentration. On the other hand, the required number of released condensed ions per individual rod becomes less on increasing the fdconcentration, as there are more rods per unit volume at 

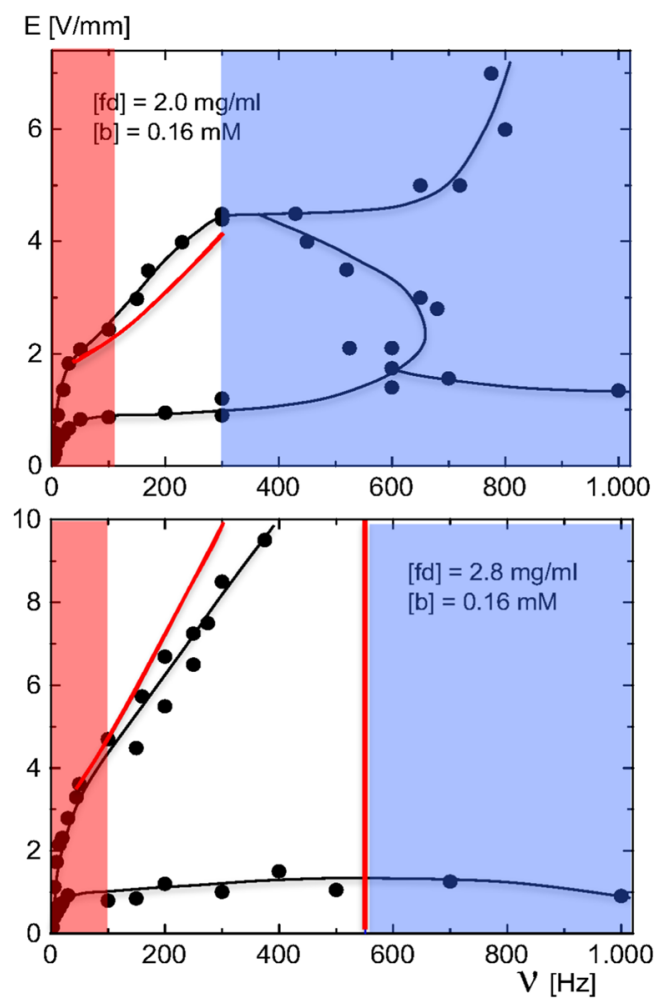

Fig. 5 The phase/state diagrams for the buffer concentration of $0.16 \mathrm{mM}$ for several fd-concentrations, as indicated in the figures. The red lines are the calculated $N^{*}$-to- $D$ transition lines, the thin black lines are guides-to-the-eye, and the vertical red line in the second figure indicates the location of the critical point. The red and blue areas are outside the validity of the theory

a higher concentration, which lowers the field amplitude where the transition occurs. As can be seen from Figs. 5 and 6 , the field amplitude where the transition occurs increases with increasing fd-concentration. The above-mentioned former mechanism wins over the latter, which is reproduced by the theory.

Since the bare charge for the two different values of the $\mathrm{pH}$ for the two buffer concentrations of 0.032 and $0.16 \mathrm{mM}$ is equal to 7800 and 8700 , respectively [24], the equilibrium number of condensed ions, in the absence of the external electric field, is found to be equal to $N_{0}-N_{c, 0}=500$ for both buffer concentrations (see Table 2). The corresponding equilibrium line-charge density is about a factor of two less than the classical predicted value from condensation theory of $e / l_{B} \approx 1100$ (where $e$ is the elementary charge and $l_{B}=0.75 \mathrm{~nm}$ is the Bjerrum length) $[33,34]$. The parameter $\alpha_{\min }$, on the contrary, is a strong function of the buffer concentration, as can be seen from Table 2. This implies that the limiting number of condensed ions on a rod with a fixed orientation under the action of an electric field strongly depends on the bulk ionic strength (see Eq. 8), in contrast to the number of condensed ions in the absence of the electric field.
Table 2 Fitted numerical parameter values for the two buffer concentrations

\begin{tabular}{|c|c|c|c|c|c|c|c|}
\hline Conc. (mM) & $N_{c, 0}$ & $\alpha_{l i m}$ & $D / D_{0}$ & $\alpha_{t h r}$ & $\mathcal{T}$ & $\mathcal{C}_{d}$ & $\tau_{\text {dif }}$ \\
\hline 0.032 & 7300 & $1.0 \times 10^{-5}$ & $1 / 350$ & - & - & - & - \\
\hline 0.16 & 8200 & $2.2 \times 10^{-4}$ & $1 / 350$ & $1 / 2$ & 65 & $10^{5}$ & 0.039 \\
\hline
\end{tabular}

\section{The dynamical behavior on approach of the $N^{*}$-to- $D$ transition line}

The remaining parameters that affect the dynamical behaviour are (i) the growth time $\mathcal{T}$ in Eq. 6 of the isotropic meta-stable state towards the nematic state, (ii) the effective dissociation constant $\mathcal{C}_{d}$ of condensed ions in Eq. 7, (iii) the
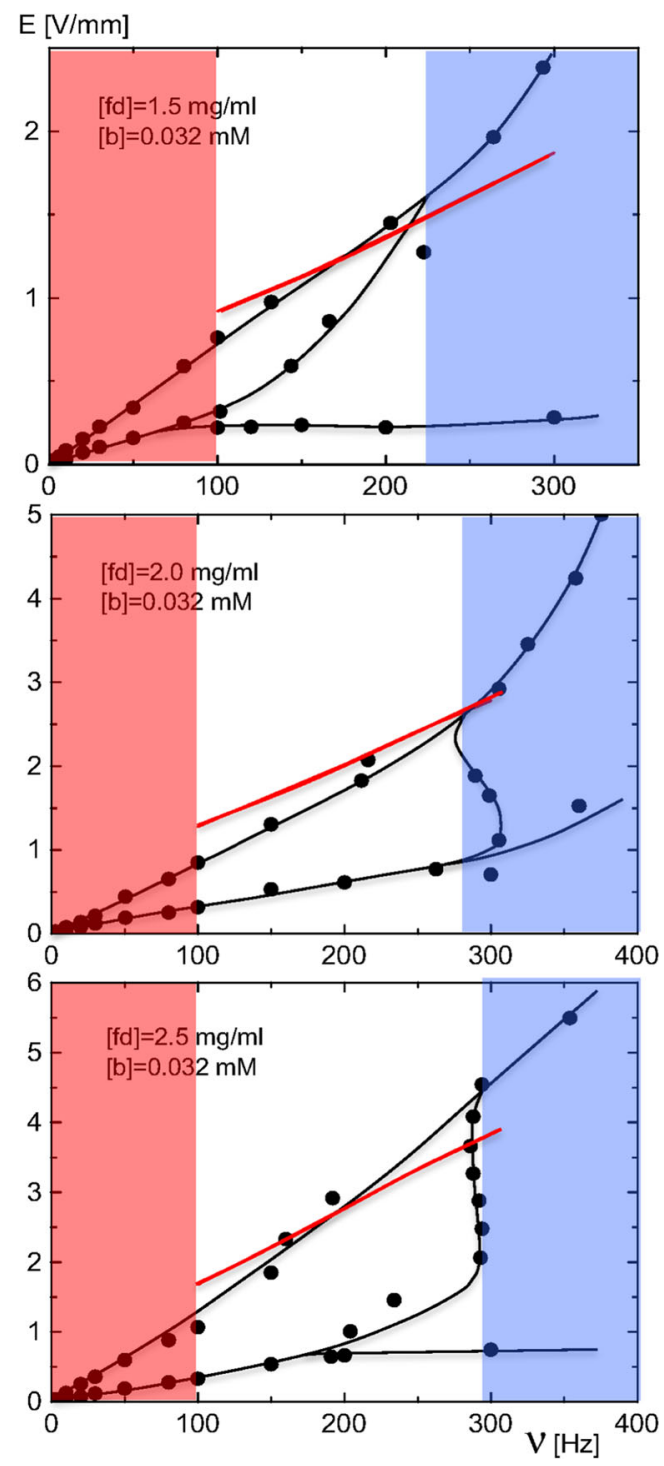

Fig. 6 The same as in Fig. 5, but now for a buffer concentration of $0.032 \mathrm{mM}$ 
time $\tau_{\text {dif }}$ that determines how long ions need to diffuse into the bulk in order to affect the overall ionic strength, and (iv) the threshold value $\alpha_{t h r}$ that sets the degree of alignment along the electric field beyond which dissociation occur. The critical exponents that quantify the divergence of the characteristic melting/forming time $\tau$ of nematic domains on approach of the $N^{*}$-to- $D$ transition line turn out to be quite independent of the precise values of $\mathbf{C}_{d}, \tau_{d i f}$, and $\alpha_{t h r}$. Reasonable agreement with experiments is found for the parameters given in Table 2 . The tabulated time for $\tau_{d i f}$ corresponds to the time required for ions in solution (with a diffusion coefficient of $2 \times 10^{-9} \mathrm{~m}^{2} / \mathrm{s}$ ) to diffuse over a distance of ten rod lengths, which seems a reasonable distance in order that dissociated condensed ions affect the bulk ionic strength. The dimensionless, numerical value of $\mathcal{T}$ was adjusted such that the limiting characteristic time of approximately $1.5 \mathrm{~s}$ far away from the transition line is reproduced by the theory. The tabulated value of $\mathcal{T}$ corresponds to a real time of $65 / D_{r}=3.3 \mathrm{~s}$ for the growth rate of a nematic domain, which is of the order of magnitude seen experimentally in fd-virus suspensions.

Figure 7 gives a comparison between the present theory with the above mentioned numerical values of the parameters and the experiments from Ref. [11], where the divergence of the characteristic time on approach of the $N^{*}$-to- $D$ transition line has been measured. Figure $7 \mathrm{a}$ is a schematic overview of the experimentally found behaviour of the characteristic time [11]: on approach of the critical point by lowering the field-amplitude at the critical frequency a power-law divergence is found with a critical exponent of $\mu_{E}=1.39 \pm 0.18$ (see the data points in Fig. $7 b$ ), while an exponent of $\mu_{v}=0.65 \pm 0.15$ is found on approach of the critical point on increasing the frequency (see Fig. 7c), and a logarithmic divergence is found for an off-critical approach of the $N^{*}$-to- $D$ transition line (as shown in Fig. 7d). The critical exponent $\mu_{\tau}^{E}$ is defined as,

$\tau-\tau_{b} \sim\left(\frac{E}{E_{c}}-1\right)^{-\mu_{\tau}^{E}}$,

for the critical divergence of the characteristic time $\tau$ on lowering the field amplitude at the critical frequency $v_{c}$ (Fig. $7 b$ ), and similarly,

$\tau-\tau_{b} \sim\left(1-\frac{v}{v_{c}}\right)^{-\mu_{\tau}^{v}}$,

on increasing the frequency at the critical field amplitude $E_{c}$ (Fig. $7 \mathrm{c}$ ). Here, $\tau_{b}$ is the non-critical background value of the characteristic time of about $1.5 \mathrm{~s}$ far away from the critical point. The theory also predicts a power-law divergence, with exponents equal to $\mu_{\tau}^{E}=0.89$ (instead of the experimental value $1.39 \pm 0.18$ ), and $\mu_{\tau}^{v}=0.91$ (instead of $0.65 \pm 0.15$ ). It was not possible to find parameters such that the experimentally found critical exponents are accurately reproduced by the theory. The differences between the theoretical predictions and the experimental results for the values of the critical exponents is most probably due to the fact that interactions between rods due to electro-osmotic flow come into play. A power-law divergence is also predicted by theory for off-critical approaches of the $D$-to- $N^{*}$ transition line, with a similar exponent 0.98 as for the critical approaches. The experiments reveal, on the contrary, a logarithmic divergence,

$\tau-\tau_{b} \sim{ }^{10} \log \left(\frac{E}{E_{\text {trans }}}-1\right)$,

where $E_{\text {trans }}$ is the location of the transition line for a fixed frequency of $150 \mathrm{~Hz}$. This discrepancy might be due to the interactions between neighboring nematic domains.
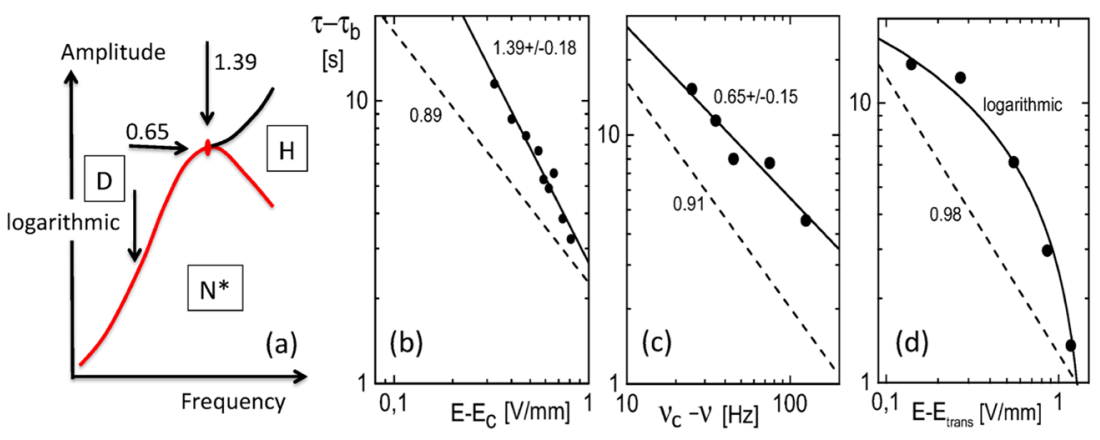

Fig. 7 The divergence of the characteristic time for melting and forming of nematic domains in the dynamical state. a A schematic of the experimentally found divergence of the characteristic time on approach of the $N^{*}$-to- $D$ transition line: a power law divergence is found on approach of the critical point (with an exponent of $1.39 \pm 0.18$ on lowering the amplitude, and $0.65 \pm 0.15$ on increasing the frequency), while a logarithmic divergence is found for an off-critical approach. b The divergence as a function of the electric-field amplitude at the critical frequency $v_{c}$, and $\mathbf{c}$ as a function of the frequency at the critical field amplitude $E_{c}$. d The divergence for the off-critical approach at a fixed frequency of $150 \mathrm{~Hz}$, where $E_{\text {trans }}$ is field strength at the transition line. The data points are taken from Ref. [11], for an fdconcentration of $2.0 \mathrm{mg} / \mathrm{ml}$, with a buffer concentration of $0.16 \mathrm{mM}$. The dashed lines are the predictions by theory. The numbers in the figures are values for the critical exponents 
In order to reproduce the experimentally found critical exponents accurately, the theory should be improved to include inter-rod interactions due to field-induced electroosmotic flow (for the critical approach), the existence of domain boundaries, and interactions between domains (for the off-critical approach).

\section{Summary and conclusions}

The phase/state behavior of concentrated suspensions of highly charged rods (fd-virus particles) have been investigated experimentally in Ref. [8], where a dynamical state (the $D$-state) is identified in which nematic domains persistently melt and form. The time scale on which melting and forming of the domains occurs is about $1.5 \mathrm{~s}$ far away from the transition line where the quiescent chiralnematic/isotropic coexistent state (the $N^{*}$-phase) transforms to the dynamical state. On approach of the $N^{*}$-to- $D$ transition line, the characteristic time for domain melting and forming is found experimentally to diverge [11]. The origin of the dynamical state is attributed to cyclic dissociation and association of condensed ions which leads to an alternating increase and decrease of the Debye screening length [9] (with a corrigendum in Ref. [10]). This in turn leads to an alternating, time-dependent effective thickness of the rods, such that the lower isotropic-nematic binodal is alternatingly crossed.This leads to the observed cyclic melting and forming of nematic domains.

There are three coupled equations of motion involved in the theory for the dynamical state: (A) an equation of motion for melting of the unstable nematic, (B) for the metastable growth of the (near-) isotropic state, and (C) for the number of condensed ions. The a priori unknown variables which enter these equations of motion are the number of condensed ions [in (A), (B), and (C)], the free diffusion coefficient of the condensed ions [in (A)], the time constant for the growth of the nematic phase [in (B)], the limiting number of condensed ions after a rod with fixed orientation is subjected for a long time to the oscillating field, the effective condensed-ion dissociation constant, and the threshold value for the orientation of a rod along the external field beyond which dissociation occurs [in (C)]. It turns out that the numerical results are relatively insensitive to the effective condensed-ion dissociation constant and the threshold value of orientation beyond which dissociation is possible. Independent of the three equations of motion, the time that is required for ions to diffuse from (or to) the layer of condensed ions into (or from) the bulk solution is an additional variable.

A few approximations are made in the theory: (i) during a cycle of the external electric field, the configuration of the rods is assumed to remain essentially unchanged, (ii) the finite size of domains is not considered, that is, the theory applies to a homogeneous system, (iii) the rodrod interactions through field-induced electro-osmotic flow are neglected, and (iv) a semi-empirical expression for the field-induced dissociation/association of condensed ions is proposed. The first assumption (i) limits the validity of the theory to frequencies larger than approximately $100 \mathrm{~Hz}$ for the fd-virus particles under consideration, while assumption (iii) limits the validity to frequencies lower than the critical frequency. Above the critical frequency polarization is essentially absent. The homeotropic $H$-phase that is formed at these higher frequencies is stabilized due to hydrodynamic interactions mediated by field-induced electro-osmotic flow.

Experimental phase/state diagrams for various fdconcentrations and ionic strengths are presented, and the location of the $D$-to- $N^{*}$ transition line is compared with the above mentioned theory $[9,10]$. In view of the approximate nature of the theory, the location of the $D$-to- $N^{*}$ transition lines in the field-amplitude versus frequency plane are reasonably well reproduced within the frequency range where the theory applies. The location of the transition lines as a function of fd-concentration and the buffer concentration (or ionic strength) are captured by the theory. Especially the subtle dependence of the location of the transition lines on the fd-concentration is captured, where there are two counter acting effects: on increasing the fd-concentration, the location of the transition line shifts to higher field strengths as the difference in concentration with the lowerbinodal concentration increases, but at the same time the field strength tends to decrease because the number of ions that should be released per rod becomes less as more rods are present. The critical divergence of the characteristic time for melting and forming of domains is also found by the theory. However, the theoretical values for critical exponents on approach of the critical point are somewhat different from those found experimentally. Moreover, for an off-critical approach of the transition line, a power-law divergence is predicted by the theory while a logarithmic divergence is observed experimentally. The former difference is most probably due to the increasing importance of interactions due to electro-osmotic flow, while the latter might be due to the interactions between domains, which are not included in the theory.

The most significant challenge to improve the theory is a quantitative description of field-induced dissociation/association of condensed ions. As mentioned above, the semi-empirical equation of motion for the number of condensed ions contains as many as three a priori unknown variables. The solution of the non-linear Poisson-Boltzmann equation for rod-like colloids in an oscillatory external electric field would not only more precisely quantify an equation of motion for the number of condensed ions but also reduce 
the number of unknown variables. Secondly, the present theory assumes a homogeneous nematic, and thus neglects the finite size of the nematic domains. The analysis of the Smoluchowski equation could possibly be extended to include spatial inhomogeneities of the orientational order parameter.

Open Access This article is distributed under the terms of the Creative Commons At tribution 4.0 International License (http:// creativecommons.org/licenses/by/4.0/), which permits unrestricted use, distribution, and reproduction in any medium, provided you give appropriate credit to the original author(s) and the source, provide a link to the Creative Commons license, and indicate if changes were made.

\section{References}

1. Fraden S, Hurd AJ, Meyer RB (1989) Phys Rev Lett 21:2373

2. Dassanayake U, Fraden S, van Blaaderen A (2000) J Chem Phys $112: 3851$

3. Yethiray A, van Blaaderen A (2003) Nature 421:513

4. Hynnine A-P, Dijkstra M (2005) Phys Rev Lett 94:138303

5. Hu Y, Glass JL, Griffith AE, Fraden S (1994) J Chem Phys 100:4674

6. Isambert H, Ajdari A, Viovy JL, Prost J (1997) Phys Rev Lett 78:971

7. Lele PP, Mittal M, Furst EM (2008) Langmuir 24(1):2842

8. Kang K, Dhont JKG (2010) Soft Matter 6:273

9. Dhont JKG, Kang K (2014) Soft Matter 10:1987

10. Dhont JKG, Kang K (2015) Soft Matter 11:2893

11. Kang K, Dhont JKG (2009) Eur Phys J E 30:333

12. Torbet J, Maret G (1981) Biopolymers 20:2657

13. Fraden S (1995) Phase Transitions in Colloidal Suspensions of Virus Particles. In: Baus M, Rull LF, Ryckaert JP (eds) Observation, Prediction and Simulation of Phase Transitions in Complex Fluids. NATO-ASI Series C, vol 460. Kluwer Academic Publishers, Dordrecht, p 113

14. Dogic Z, Fraden S (2000) Langmuir 16:7820

15. Dogic Z, Fraden S (2001) Philos Trans R Soc A 359:997

16. Grelet E, Fraden S (2003) Phys Rev Lett 90:198302

17. Purdy KR, Fraden S (1703) Phys Rev E: Stat, Nonlinear, Soft Matter Phys 70(06):2004

18. Tombola F, Ferrarini A, Grelet E (2006) Phys Rev Lett 96:258302

19. Dogic Z, Fraden S (2006) Curr Opin Colloid Interface Sci 11:47

20. Grelet E, Lettinga MP, Bier M, van Roij R, van der Schoot P (2008) J Phys Condens Matter 20:494213

21. Lettinga MP, Dhont JKG, Zhang Z, Messlinger S, Gompper G (2010) Soft Matter 6:4556

22. Pouget E, Grelet E, Lettinga MP (1704) Phys Rev E: Stat, Nonlinear, Soft Matter Phys 84(04):2011

23. Naderi S, Pouget E, Ballesta P, Grelet E, van der Schoot P, Lettinga MP (2013) Phys Rev Lett 111:037801
24. Zimmermann K, Hagedorn J, Heuck CC, Hinrichsen M, Ludwig J (1986) J Biol Chem 261:1653

25. Kang K, Wilk A, Patkowski A, Dhont JKG (2007) J Chem Phys 126:214501

26. Onsager L (1942) Phys Rev 62:558

27. Onsager L (1949) Ann N Y Acad Sci 51:627

28. Lekkerkerker HNW, Vroege GJ (1993) Philos Trans R Soc A $344: 419$

29. Stroobants A, Lekkerkerker HNW, Odijk Th (1986) Macromolecules 19:2232

30. Odijk Th (1990) J Chem Phys 93:5172

31. Dhont JKG, Kang K (2011) Eur Phys J E 34:40

32. Bradac Z, Kralj S, Zumer S (1705) Phys Rev E: Stat, Nonlinear, Soft Matter Phys 65(02):2002

33. Manning GS (1996) Ber Bunsen-Ges Phys Chem Chem Phys 100:909

34. Manning GS (2007) J Phys Chem B 111:8554

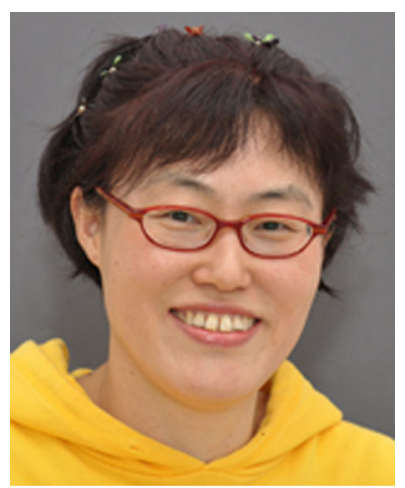

Kyongok Kang had a PhD in Physics at Kent State University, and a postdoctoral fellowship in the Institute of Complex Systems at the Research Center in Juelich, Germany. Since 2007, she is a permanent scientist. She investigates both non-equilibrium phase transitions and equilibrium orientation dynamics of systems of charged chiral rods. She developed instrumentation to investigate nonequilibrium phases/states, by image-time correlation, small angle dynamic light scattering, and electric birefringence.

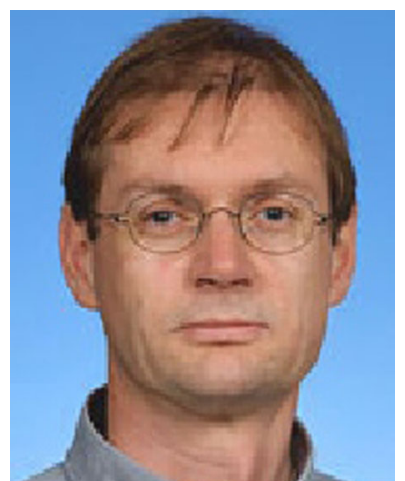

Jan Dhont was appointed as an associated professor at the van 't Hoff laboratory, Utrecht University, in the group of Prof. H.N.W. Lekkerkerker in 1987. In 2000, he was appointed as a director at the Research Center of Juelich, and in 2001 as a full professor at the Heinrich-Heine-University in Duesseldorf. His current research interests are concerned with the non-equilibrium behavior of soft-matter systems in external fields, such as flow, confinement, and electric fields. 